\title{
STATUS OF HIGH POLARIZATION DC HIGH VOLTAGE GALLIUM ARSENIDE PHOTOELECTRON GUNS*
}

\author{
M. Poelker\#, P. Adderley, J. Brittian, J. Clark, J. Grames, J. Hansknecht, J. McCarter, M.L. \\ Stutzman, R. Suleiman, K. Surles-Law, Jefferson Lab, 12000 Jefferson Ave., Newport News, VA \\ 23606 U.S.A.
}

\section{Abstract}

Users receive very high beam polarization from reliable GaAs photoelectron guns at facilities worldwide. Satisfaction with beam quality (and a number of lab closures) has reduced the level of polarized source $R \& D$ from the heyday of 1990s. However, new experiments and new accelerators proposals including high current unpolarized machines, require GaAs photoguns with capabilities that exceed today's state of the art. This submission describes the capabilities of today's highpolarization DC high voltage GaAs photoguns and discusses issues that must be addressed to meet new demands.

\section{INTRODUCTION}

Since the invention of the first spin-polarized GaAs photoelectron gun in the mid-1970s, numerous experimental programs have grown from this technological advance. The challenges associated with meeting today's accelerator applications have largely been solved, with users receiving highly polarized beam (>80\%) from reliable DC high voltage GaAs photoguns for long periods of time. Ironically, user satisfaction, and regrettably numerous laboratory closures, has reduced the amount of R\&D devoted to polarized source development. Today, only three dedicated user facilities continue to provide polarized electron beams on a daily basis (Table1). Recently however, compelling new experiments such as Qweak at CEBAF/Jefferson Lab, and ambitious new accelerator proposals such as the International Linear Collider and Electron Ion Collider, call for polarized beam with specifications exceeding today's state of the art. In addition, very high current unpolarized accelerators require beam from GaAs photoguns. These developments necessitate a renewed R\&D program dedicated toward better appreciating the limitations of today's GaAs photoguns.

This paper describes the state of the art of polarized electron source technology largely in the context of the CEBAF program (i.e., CW, $\sim 200 \mu \mathrm{A}$ maximum average current), however many of the features of CEBAF polarized GaAs photoguns are shared by those at other labs, including pulsed and unpolarized machines. A recent publication [1] described the CEBAF polarized source program through 2002. Since then, a number of interesting developments have occurred related to photocathodes, drive lasers, appreciation for vacuum, load locked gun development and high current lifetime studies.

*Notice: Authored by Jefferson Science Associates, LLC under U.S DOE Contract No. DE-AC05-06OR23177.

\#poelker@jlab.org
Table 1: Accelerator-based polarized electron sources, past and present. Shaded entries indicate diminished programs and/or facilities that no longer provide polarized electron beam to users.

\begin{tabular}{|c|c|c|}
\hline Facility & Beam Structure & Gun Voltage \\
\hline Bonn-ELSA & Pulsed & $50 \mathrm{kV}$ \\
\hline CEBAF & $\mathrm{CW}$ & $100 \mathrm{kV}$ \\
\hline Mainz Microtron & $\mathrm{CW}$ & $100 \mathrm{kV}$ \\
\hline MIT-Bates & Pulsed & $60 \mathrm{kV}$ \\
\hline Nagoya/JLC & Pulsed & $200 \mathrm{kV}$ \\
\hline NIKHEF & Pulsed & $100 \mathrm{kV}$ \\
\hline SLAC/SLC & Pulsed & $120 \mathrm{kV}$ \\
\hline
\end{tabular}

\section{COMMERCIAL STRAINED- SUPERLATTICE PHOTOCATHODE}

A dramatic recent development has been the commercialization of a strained-superlattice GaAs photocathode by SVT Associates, together with SLAC, as part of a highly successful SBIR program [2]. This new material consistently provides $85 \%$ polarization and $\mathrm{QE} \sim$ $1 \%$, significantly beyond the capability of the only other commercially available high polarization photocathode. Commercial availability of high polarization photocathode material is very important for user-based accelerators, ensuring a reliable and consistent long-term supply.

A sample of strained-superlattice photocathode material from SVT Associates was in constant use at CEBAF between September, 2005 and April, 2007, providing beam current up to $200 \mu \mathrm{A}$ and $85 \%$ polarization, with users enjoying a $38 \%$ increase in figure of merit $\left(\mathrm{P}^{2} \mathrm{I}\right)$ compared to previous conditions using stained-layer GaAs and $75 \%$ polarization. Over this period, approximately 1000 Coulombs were extracted and the photocathode sample was heated and reactivated a total of seven times, with maximum QE gradually dropping from $0.7 \%$ to $0.4 \%$, yet polarization remained essentially constant at $85 \%$.

Note however, initial experience with this material was disappointing, with QE and polarization values significantly lower than reported by SLAC. Over time it became clear that the edge-anodizing procedure, which is critical for successful long lifetime, high average current beam delivery at CEBAF, needed modification [3]. 
Edge anodizing limits the photocathode active area to just the center portion of the photocathode $(5 \mathrm{~mm}$ diameter versus $13 \mathrm{~mm}$ total). An anodized edge eliminates the possibility of photoemission from photocathode locations where electrons can travel extreme trajectories, striking the anode plate or beampipe walls, liberating gas and hastening QE decay. Historically, photocathode samples were always cleaned with atomic hydrogen following the edge anodizing process, to remove contaminants that might have been introduced onto the sample during repeated rinses with hydrocarbon solvents and water. It became clear that hydrogen cleaning, at least in the manner practiced at CEBAF/Jefferson Lab, seriously degraded strained superlattice performance [3]. Eventually, a new anodization procedure was developed that greatly reduced the level of contamination introduced onto the photocathode. In addition, the vendor successfully applied a protective arsenic cap to the material. As a result, edge-anodized samples now provide high $\mathrm{QE}$ and polarization, without requiring hydrogen cleaning.

\section{FIBER-BASED DRIVE LASER}

An indirect benefit of using strained-superlattice photocathodes from SVT Associates came with the realization that fiber-based telecommunications lasers and amplifiers at $1560 \mathrm{~nm}$ could be frequency doubled to provide required light at $780 \mathrm{~nm}$ [4]. The main advantages of a fiber-based drive laser are three-fold: 1) fiber-based laser systems have no mirrors to clean or align, 2) fiber-coupled diode lasers can be gain switched to obtain high repetition rate, phase-stable optical pulse trains without feedback loops, and 3) relatively inexpensive "off the shelf" fiber-based amplifiers can boost average optical power well above levels of modelocked Ti-Sapphire lasers. Fiber-based laser systems now generate all of the beams produced at CEBAF (one laser for each experimental hall). The move to fiber-based drive lasers has significantly reduced accelerator downtime associated with maintenance of the modelocked Ti-Sapphire lasers that were used previously, when $850 \mathrm{~nm}$ light was required for high polarization.

Although not relevant to polarized electron beam production, a green-light fiber laser system also has been constructed, via frequency doubling at $1064 \mathrm{~nm}$ (another popular telecommunications wavelength). The green light fiber-based drive laser will be used for high current unpolarized beam experiments similar to those described below. In addition, there are plans to construct a more powerful version using "off the shelf" amplifiers to obtain at least $25 \mathrm{~W}$ at $532 \mathrm{~nm}$ for a Compton polarimeter at experimental Hall C. Numerous recent publications suggest even higher power green-light systems (>100W) are possible using fiber technology [5].

\section{VACUUM STUDIES}

Over the course of years, it became clear that measured vacuum pressure within CEBAF photoguns and test chambers always exceeded predictions based on the simple equation below, using vendor-quoted pump speeds and commonly accepted values for outgassing rates. To address this discrepancy and better appreciate the vacuum limitations of modern photoguns, tests were conducted to quantify outgassing rate and non-evaporable getter (NEG) pump speed [6].

$$
P_{\text {ultimate }}=\frac{\text { OutgasRate } \bullet \text { SurfaceArea }}{\text { PumpSpeed }}
$$

\section{Outgassing Rate}

The outgassing rates of 304 stainless steel vacuum chambers were measured, comparing a variety of chemical and heat treatments. Measurements indicate essentially identical outgassing rates for each chamber, $1 \times 10^{-12}$ TorrL/seccm ${ }^{2}$, a value considerably higher than remarkably low values frequently reported in literature but not dissimilar from often-quoted industry standards [7]. Figure 1 indicates that electropolishing and vacuum firing, as per the American Vacuum Society recommended procedure [8], produces a slightly better outgassing rate, and required fewer bakeouts to achieve this value.

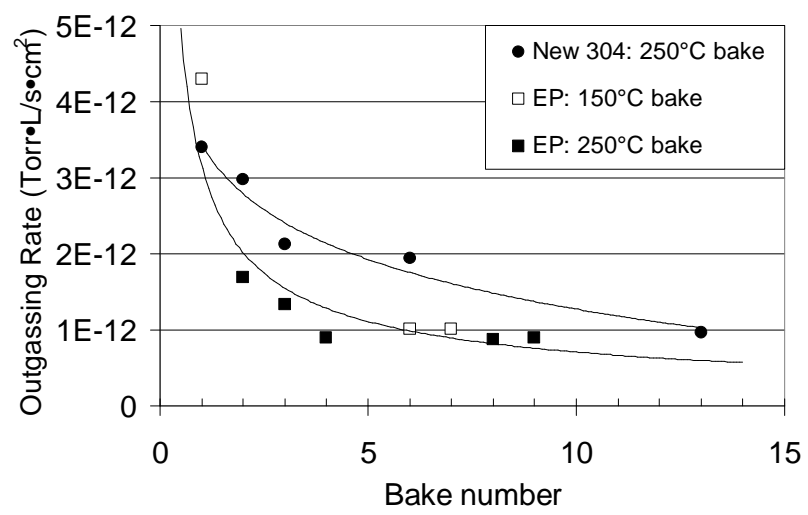

Figure 1. Outgassing rate of a two gun vacuum chambers made of 304 stainless steel. One chamber (squares) received no special pretreatment, the other chamber (circles) was electropolished and vacuum fired.

\section{NEG Pump Speed Measurements}

The dominant gas species in modern UHV GaAs photoguns is hydrogen and NEG pumps provide most of the pumping, with ion pumps managing inert gasses like helium and methane. Vendor SAES Getters recommends resistive activation of NEG pumps using electrical current passed through the NEG pump substrate material. This type of activation however, produces a large gas load and high pressure, which can potentially contaminate the 
photocathode surface. As such, NEG pump activation within the vent/bake guns at CEBAF occurs passively via the $250^{\circ} \mathrm{C} / 3$ hour bakeout. The pump speed measurements shown in Figure 2 indicate passive activation provides only $\sim 40 \%$ of the pumping compared to resistive activation as per vendor recommendation.

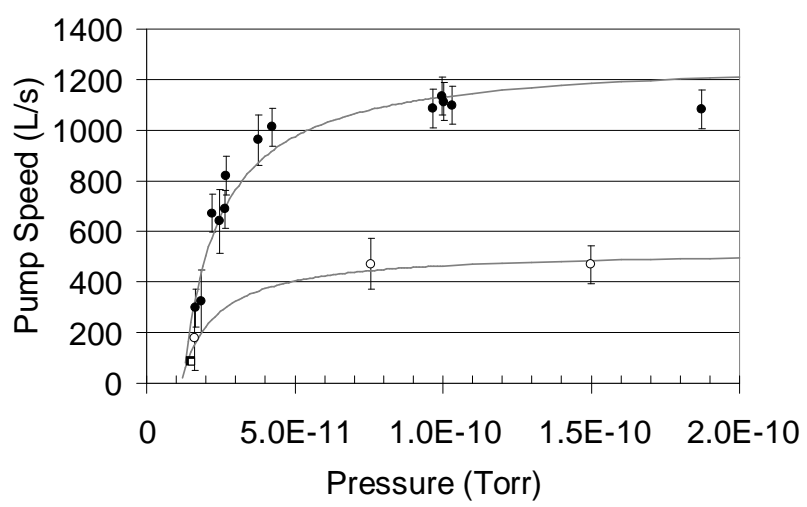

Figure 2. Pump speed measurements versus pressure for a single SAES WP950 getter module: full activation through resistive heating (closed circles) and passive activation via chamber bakeout (open circles).

Another interesting feature of the plots in Figure 2 is the pump speed roll-over at low pressure, behavior that is difficult to interpret and not described in literature. The data follow the characteristic trend described by

$$
S=S_{\max }\left(1-\frac{P_{o}}{P}\right)
$$

where $S_{\max }$ is maximum pump speed and $P_{o}$ is the system base pressure. Data could be reproduced in successive trials, indicating the NEG pumps were not saturated. Interestingly, both NEG activation protocols (active and passive activation) produced nearly the same base pressure: $\sim 1.2 \times 10^{-11}$ Torr. It is possible the UHV extractor gauges used for these measurements are not accurate below $\sim 5 \times 10^{-11}$ Torr, well above the vendorstated pressure monitoring capability [9]. However, it is well known that low pressure measurements with hot filament gauges are difficult, with accuracy subject to gauge calibration, gauge placement relative to the chamber walls, orientation and prior history [10].

\section{In-house NEG coating}

Pump speed measurements were made of in-house manufactured NEG coating applied to the inner surface of a photogun vacuum chamber, as per reference 11 . Figure 3 indicates the entire coating provides $\sim 200 \mathrm{~L} / \mathrm{s}$ pumping (at higher pressures), giving a modest pump speed per unit area of $\sim 0.02 \mathrm{~L} / \mathrm{s} \cdot \mathrm{cm}^{2}$. Composition analysis indicates $25 \%$ titanium, $50 \%$ vanadium and $25 \%$ zirconium. Enhanced pump speed might be obtained using magnetron sputtering techniques and/or replacing argon gas with krypton [12].

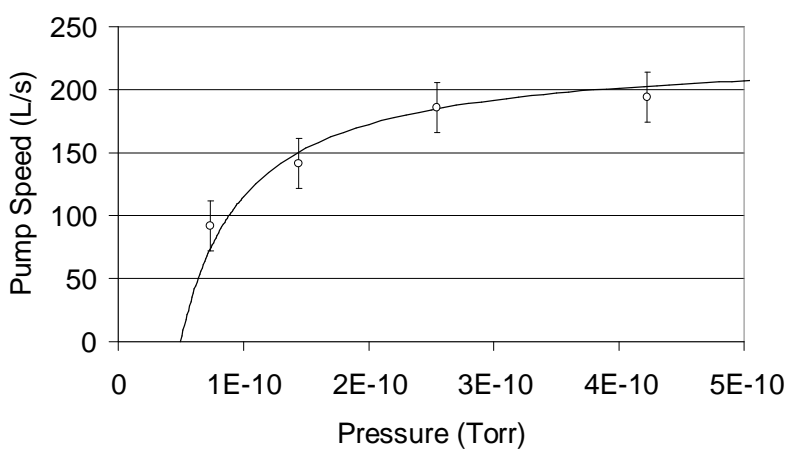

Figure 3. Pump speed of NEG coating.

\section{LOAD-LOCKED GUN}

Vent/bake photoguns have supplied the electrons at CEBAF since 1996. To a great extent, these guns have operated so reliably that there has been little incentive to replace them with a load locked gun. However, a new parity violation experiment, Qweak [13], requiring $180 \mu \mathrm{A}$ average current for one year duration has received approval for beamtime. Past experience with parity violation experiments indicates the importance of operating under constant, stable conditions, with photocathode QE decaying very slowly. At $180 \mu \mathrm{A}$ for Qweak, combined with simultaneous beam delivery to other halls, over 20 Coulombs will be extracted from the gun each day, roughly a factor of two more than usual. Successful completion of this high-profile experiment necessitates improved photogun charge lifetime and the ability to quickly replace the photocathode sample when necessary. This provides the motivation for installing a load locked gun at CEBAF.

The first CEBAF load locked gun was described at a previous particle accelerator conference [14]. Since then, a new load locked gun has been constructed, incorporating vacuum improvements described above (electropolishing and vacuum firing, reduced surface area, and NEG coating of internal vacuum surfaces) as well as design improvements that improve gun reliability and operation (e.g., bellows were added between each chamber, pucks do not have opportunity to roll). In addition, the preparation chamber was modified to hold four photocathode samples and an activation mask provides the means to selectively activate only the center of each photocathode, eliminating the necessity of edge anodization. Finally, a "suitcase" approach was adopted, to keep the gun footprint small, allowing relatively rapid replacement of all photocathode samples, requiring a relatively simple bakeout of a small intermediate vacuum chamber. 


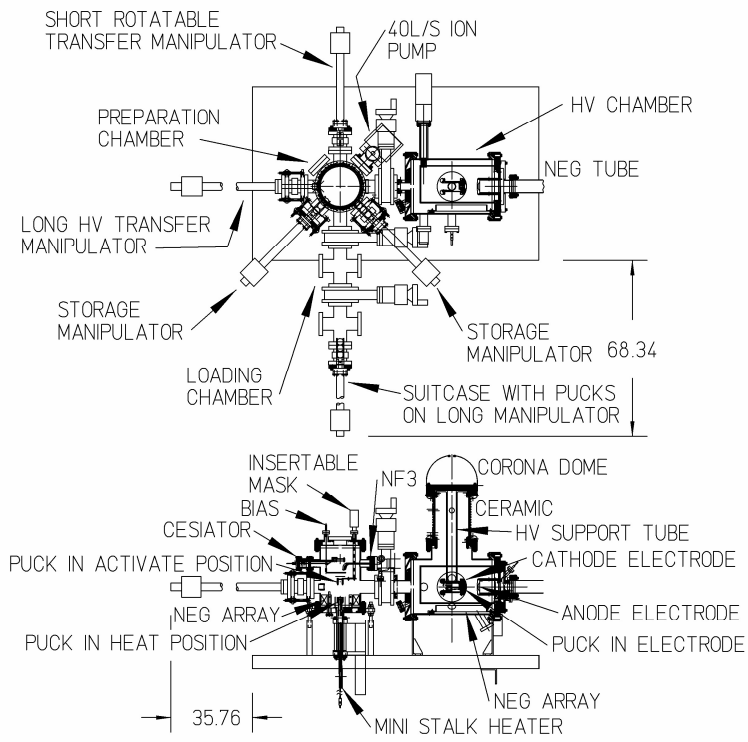

Figure 4. Schematic of the new CEBAF load locked gun to be installed during the 2007 summer shutdown.

\section{LIFETIME STUDIES}

Photocathode lifetime of modern DC high voltage GaAs photoguns is limited primarily by ion backbombardment, the mechanism where residual gas at the cathode/anode gap is ionized by the extracted electron beam and back-accelerated toward the photocathode. A very simple model suggests the possibility of improving photogun lifetime by simply increasing the size of the drive laser beam at the photocathode. For a large laser spot, total ion production at the cathode/anode gap remains the same, but ion damage would be distributed over a larger area and therefore $\mathrm{QE}$ at specific photocathode locations degrades more slowly. Lifetime enhancement, per this simple model, would be equal to the ratio of the two laser beam areas.

The new CEBAF load locked gun was used to test this idea. Measurements were made using bulk GaAs and green light at DC beam currents between 1 and $10 \mathrm{~mA}$, and laser spot sizes of $320 \mu \mathrm{m}$ and $1550 \mu \mathrm{m}$ (FWHM) [15]. Indeed, lifetime was observed to increase using a larger laser spot (Figure5), however not by the ratio of the two laser beam areas. Lifetime scaled by a factor of $\sim 5$ whereas the simple model predicted an enhancement of 18. This is not too surprising, considering that ion damage is not uniformly distributed across the photocathode surface: ions will be focused toward the electrostatic center of the photocathode. Furthermore, the ionization cross section differs for each residual gas species and this cross section varies with electron beam energy, from $0 \mathrm{~V}$ at the photocathode surface to $100 \mathrm{kV}$ at the anode. In addition, the stopping depth of each ion within the material varies as a function of gas species and energy. Still, this measurement demonstrates remarkably high charge lifetime (>1000 C) at average beam currents above $1 \mathrm{~mA}$, and marks an important step toward appreciating the complicated factors that limit photocathode lifetime.

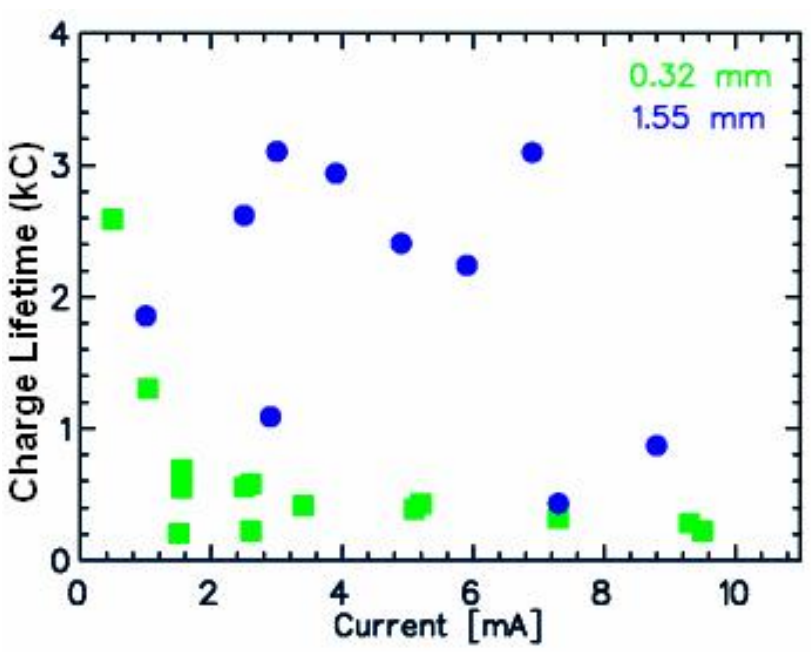

Figure 5. Charge lifetime measurements as a function of average beam current, using the new CEBAF load locked gun and different laser spot sizes. Lifetime enhancement was obtained using a larger laser spot at the photocathode.

\section{CONCLUSIONS}

Polarized electron sources can comfortably meet the demands of the today's accelerator/physics programs, however, the beam current demands of future accelerators such as the ILC and the EIC exceed today's state of the art. In a broad sense, three parameters determine the length of time that a photogun can operate at a specified beam current: vacuum pressure, maximum available laser power, and photocathode QE. Each of these topics receives $R \& D$ attention at CEBAF/Jefferson Lab with the end-goal focused on the production of high polarization and high average current beyond $1 \mathrm{~mA}$, to support EIC preliminary design efforts. Fiber-based laser systems appear to meet high average current requirements, however photocathode cooling will need to be addressed when laser power exceeds $\sim 1 \mathrm{~W}$. Spintronics photocathodes such as halfmetal $\mathrm{CrO}_{2}$ [16] may provide high polarization with $\mathrm{QE}$ exceeding $1 \%$. This possibility is the main thesis topic of one of the authors. In keeping with higher beam current operation, it is time to reevaluate the cathode/anode design of the CEBAF photogun, to better understand the nature of beam loss and the empirical observation that photoemission from the edge of the photocathode hastens QE decay. As part of this effort, the cathode/anode optic will be redesigned with a smaller gap, which should provide longer lifetime because there will be fewer ions within the gap to damage the photocathode. Vacuum improvements will likely require improved vacuum gauging, and modest effort has been directed toward constructing the improved Helmer gauge used for pressure measurement at $10^{-15}$ Torr at CERN [17]. Finally, this submission did not address the topic of charge limit [18], the phenomenon related accumulation of trapped charge at the surface of the photocathode, which serves to reduce $\mathrm{QE}$ at high laser powers. Historically, this has been most problematic for 
pulsed-machines operating at $\mathrm{nC}$ bunch charge, but tomorrow's high average current $\mathrm{CW}$ machines will also suffer from this effect, particularly as the photocathode becomes damaged due to ion backbombardment.

\section{REFERENCES}

[1] C. K. Sinclair, P. A. Adderley, B. M. Dunham, J. C. Hansknecht, P. Hartmann, M. Poelker, J. S. Price, P. M. Rutt, W. J. Schneider, and M. Steigerwald, Phys. Rev. ST Accel. Beams 10, 023501 (2007).

[2] T. Maruyama, D.-A. Luh, A. Brachmann, J. E. Clendenin, E. L. Garwin, S. Harvey, J. Jiang, R. E. Kirby, A.M. Moy, R. Prepost, and C. Y. Prescott, Appl. Phys. Lett. 85, 2640 (2004). Material can be purchased from SVT associates, Inc., 7620 Executive Drive, Eden Prairie, MN 55344

[3] M. Baylac, P. Adderley, J. Brittian, J. Clark, T. Day, J. Grames, J. Hansknecht, M. Poelker, M. Stutzman, A. T. Wu, and A. S. Terekhov, Phys. Rev. ST Accel. Beams $\underline{8} 123501$ (2005)

[4] J. Hansknecht and M. Poelker, Phys. Rev. ST Accel. Beams 9, 063501 (2006).

[5] A. Liu, M. Norsen and R. Mead, Opt Lett., 30 (2005) p. 67-69.; Dupriez, A. Piper, A. Malinowski, J. K. Sahu, M. Ibsen, Y. Jeong, L. M. B. Hickey, M. N. Zervas, J. Nilsson and D. J. Richardson, IEEE Phot. Tech. Lett., 18 (2006) 1013.
[6] M.L. Stutzman, P. Adderley, J. Brittian, J. Clark, J. Grames, J. Hansknecht, G.R. Myneni, M. Poelker, Nucl. Instrum. Meth. A, 574 (2007) p. 213-220

[7] J.F.O'Hanlon, A User's Guide to Vacuum Technology, $2^{\text {nd }}$ ed. (Wiley, New York, 1989), p. 444.

[8] Y.T. Sasaki, J. Vac. Sci. Technol. A 9 (1991) 2025.

[9] Leybold extractor gauge, model IE 514 with IONIVAC IM 520 controller.

[10] P.A.Redhead, J. Vac. Sci. Technol. A 12 (1994) 904; P.A.Redhead, Vacuum 53 (1999) 137.

[11] C Benvenuti et al., Vacuum 53 (1999) 219.

[12] Yulin Li, in Proceedings of the 2003 Particle Accelerator Conference, p. 620.

[13] Experiment described at http://www.jlab.org/qweak/

[14] W. Schneider, et.al., in Proceedings of the 1999 Particle Accelerator Conference, p. 1991.

[15] M. Poelker and J. Grames, in Proceedings of the 2005 XIth International Workshop on Polarized Sources and Targets, Tokyo, Japan.; J. Grames et al., to appear in Proceedings of the 17 International Spin Symposium, Kyoto, Japan (2006).

[16] P.A.Dowben and R. Skomski, J. Appl. Phys., 95 (2004) 7453, and references within.

[17] Helmer, J.C., Vacuum 51, sept 1998, p 7-10

[18] T. Maruyama, et. al, in Proceedings of the $14^{\text {th }}$ International Spin Symposium, Osaka, Japan (2000), and references within. 